\title{
Measuring Vascular Recovery Rate After Exercise ${ }^{\dagger}$
}

\author{
Halil Dijab, Jordi Alastruey (10) and Peter H. Charlton *(i) \\ Department of Biomedical Engineering, School of Biomedical Engineering and Imaging Sciences, King's College \\ London, King's Health Partners, St Thomas' Hospital, London SE1 7EH, UK; hd16aax@herts.ac.uk (H.D.); \\ jordi.alastruey-arimon@kcl.ac.uk (J.A.) \\ * Correspondence: peter.charlton@kcl.ac.uk \\ + Presented at the 5th International Electronic Conference on Sensors and Applications, 15-30 November 2018; \\ Available online: https: / / ecsa-5.sciforum.net.
}

Published: 14 November 2018

\begin{abstract}
The rate at which an individual recovers from exercise is known to be indicative of cardiovascular risk. It has been widely shown that the reduction in heart rate immediately after exercise is predictive of mortality. However, little research has been conducted into whether the time taken for the blood vessels to return to normal is also indicative of risk. In this study, we present a novel approach to assess vascular recovery rate (VRR) using the photoplethysmogram (PPG) signal, which is monitored by smart wearables. The VORTAL dataset (http:/ / peterhcharlton.github.io/ RRest/) was used for this study, containing PPG signals from 39 healthy subjects before (baseline) and after exercise. 31 VRR indices were extracted from the PPG pulse wave shape, as well as heart rate for comparison. The rate at which indices returned to baseline after exercise was quantified, and the consistency of changes between subjects was assessed statistically. Many VRR indices exhibited changes after exercise which were consistent between subjects. Indices derived from the timings and second derivative of pulse waves were identified as candidates for future work. The rate at which the indices returned to baseline differed between indices and subjects, indicating that they may provide additional information beyond that of heart rate, and that they may be useful for stratifying subjects. This study demonstrated the feasibility of assessing VRR after exercise from the PPG. Future studies should investigate whether VRR indices are associated with cardiovascular fitness, and the potential utility of incorporating the indices into wearable sensors.
\end{abstract}

Keywords: wearable sensors; arterial stiffness; photoplethysmogram; exercise; heart rate recovery

\section{Introduction}

The rate at which the body recovers from exercise is known to be indicative of cardiovascular risk. It has been widely shown that the rate at which the heart rate recovers immediately after exercise is predictive of cardiovascular events and all-cause mortality [1]. Indeed, heart rate recovery (the number of beats per minute by which the heart rate falls one minute after cessation of exercise) has recently been included in recommendations for cardiopulmonary exercise testing [2]. However, little research has been conducted into whether the time taken for the blood vessels to return to normal, termed the vascular recovery rate (VRR), is also indicative of risk. If the VRR could be easily measured using wearable sensors, then it could be useful for assessing health and fitness.

Many smart watches and fitness bands measure the photoplethysmogram (PPG) signal [3]. The PPG is the amount of light either reflected from or transmitted through an illuminated tissue bed. Wrist-worn devices usually obtain the PPG signal by illuminating the skin at the wrist using a light-emitting diode and measuring the amount of light reflected from the skin using a photodetector. The resulting PPG signal exhibits a pulse wave for each heartbeat, as shown in Figure 1. The pulse waves are primarily caused by the expansion and relaxation of blood vessels due to the increase and 
decrease in blood pressure with each heartbeat. Consequently, pulse wave shape is influenced by both the ejection of blood from the heart and the mechanical properties of the blood vessels.

(a)

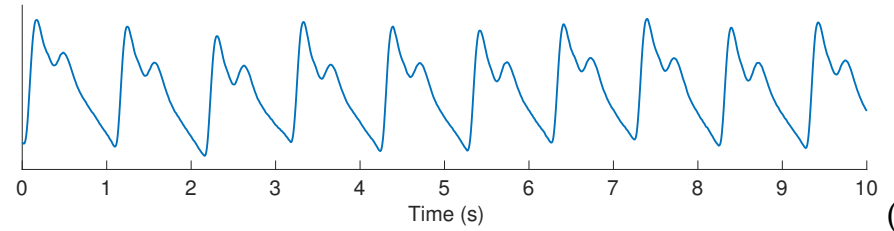

(b)

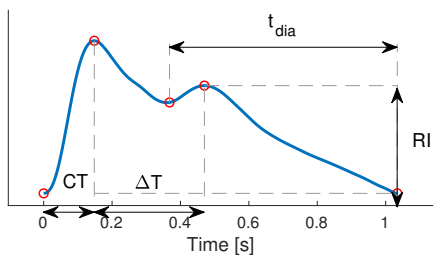

Figure 1. The photoplethysmogram (PPG) signal: (a) A 10-s recording, exhibiting a pulse wave for each heart beat-approximately one per second; (b) Extracting four exemplary vascular recovery rate (VRR) indices from a pulse wave (defined in Abbreviations section at end). au: arbitrary units

Previous research indicates that the stiffness of arteries changes during exercise, and these changes persist for several minutes afterwards [4]. The changes may be beneficial to health and fitness as they reduce the work required for the heart to pump blood around the body [5]. The effects of these changes on pulse wave shape have been investigated in $[5,6]$. However, to our knowledge no tool has been developed for analyzing PPG pulse waves to derive a measure of the VRR.

The aim of this study was to investigate the feasibility of measuring VRR indices from the PPG. Several measurements of pulse wave shape were extracted from a dataset of PPG signals acquired before and after exercise. The inter-subject consistency of changes in VRR indices during recovery from exercise, and the rate at which indices returned to baseline values, were assessed. If this approach is found to provide useful indicators of cardiovascular health, then it could be incorporated into wearable sensors for use in both everyday and clinical settings.

\section{Methods}

\subsection{Dataset of Photoplethysmogram (PPG) Signals Acquired before and after Exercise}

The VORTAL dataset contains PPG signals acquired in a controlled laboratory setting. Signals were acquired from 39 young, healthy subjects while lying down for approximately $10 \mathrm{~min}$ both before and after exercise, as described in [7]. The PPG signals were measured using an MLT1020FC finger clip infrared reflection plethysmograph and digitized at $500 \mathrm{~Hz}$. The exercise consisted of running on a treadmill until $30 \mathrm{~s}$ after the heart rate (HR) reached $85 \%$ of the age-predicted maximum.

\subsection{Extracting Vascular Recovery Rate (VRR) Indices from the PPG}

VRR indices were extracted from the PPG as follows. PPG signals were band-pass filtered to eliminate very low and very high frequency content, using $-3 \mathrm{~dB}$ cut-offs of 0.07 and $16.75 \mathrm{~Hz}$. Signal quality was assessed for consecutive $10 \mathrm{~s}$ signal segments using the approach in [8]. Low quality segments were excluded from the analysis. Pulse waves were identified using the algorithm described in [9]. Pulse wave analysis techniques were used to extract 31 vascular stiffness indices (termed VRR indices) and HR from each PPG pulse wave, as described in [10]. These time series were median filtered to attenuate high frequency variations (using a filter of order 15 beats).

\subsection{Identifying Significant Changes in VRR Indices}

The Wilcoxon Rank Sum test was used to identify any statistically significant changes in VRR indices during recovery from exercise (at the $5 \%$ significance level). This was performed by comparing the initial and final $5 \%$ of beats in the recovery recording. Two tests were performed for each VRR: one to identify a positive change, and one to identify a negative change. 


\subsection{Quantifying Changes in VRR Indices After Exercise}

Changes in VRR indices immediately after exercise were assessed as follows. The mean values of each VRR index during the initial and final $5 \%$ of beats in the recovery recording were calculated $\left(\overline{V R R}_{i, \text { init }}\right.$ and $\left.\overline{V R R}_{i, \text { final }}\right)$. The baseline value of each VRR index was calculated as its mean value during the recording at rest prior to exercise $\left(\overline{V R R}_{i, b a s e}\right)$. The percentage change in each VRR index $\left(V R R_{i}\right)$ per minute was calculated using (where $T$ is the duration of the recovery recording in minutes):

$$
\text { Percentage change in } V R R_{i}\left[\% \mathrm{~min}^{-1}\right]=100 \times \frac{\overline{V R R}_{i, \text { final }}-\overline{V R R}_{i, \text { init }}}{\left|\overline{V R R}_{i, \text { init }}-\overline{V R R}_{i, \text { base }}\right|} \times \frac{1}{T} .
$$

\section{Results and Discussion}

\subsection{Exemplary Changes in VRR Indices after Exercise}

Figure 2 shows exemplary changes in selected VRR indices after exercise for one subject. In this example the duration of systole ( $t_{s y s}$, shown in Figure $2 a$ ) increased over the first five minutes of the post-exercise recording and plateaued at a slightly higher value than the baseline value. In contrast, the ageing index (AGI) and augmentation index (AI) in Figure $2 b, c$ decreased after exercise, moving further from their baseline values. Previous studies have also found that AI remains below its baseline value for $10 \mathrm{~s}$ to hours after exercise. Finally, the HR (shown in Figure 2d), almost returned to its baseline value during the $10 \mathrm{~min}$ after exercise. Most of the change in HR occurred within the first two minutes, as opposed to the five minutes for $t_{\text {sys }}$. This indicates that even though both these indices are based on timings of the pulse wave, the mechanisms responsible for their changes may differ. Some high frequency variation in indices was still present, despite the use of median filtering, indicating that in the future it may be useful to adopt stricter signal quality criteria when selecting which pulse waves to include in the analysis. The VRR indices had not returned to baseline values by the end of the recording, indicating that future studies should record signals for longer than 10 min post-exercise (which is supported by [4]).

(a)
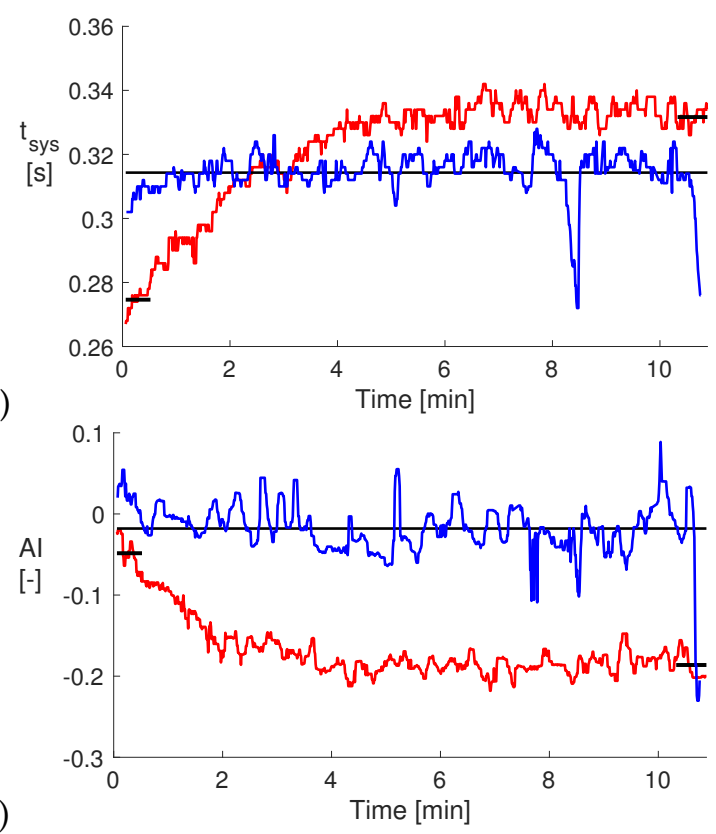
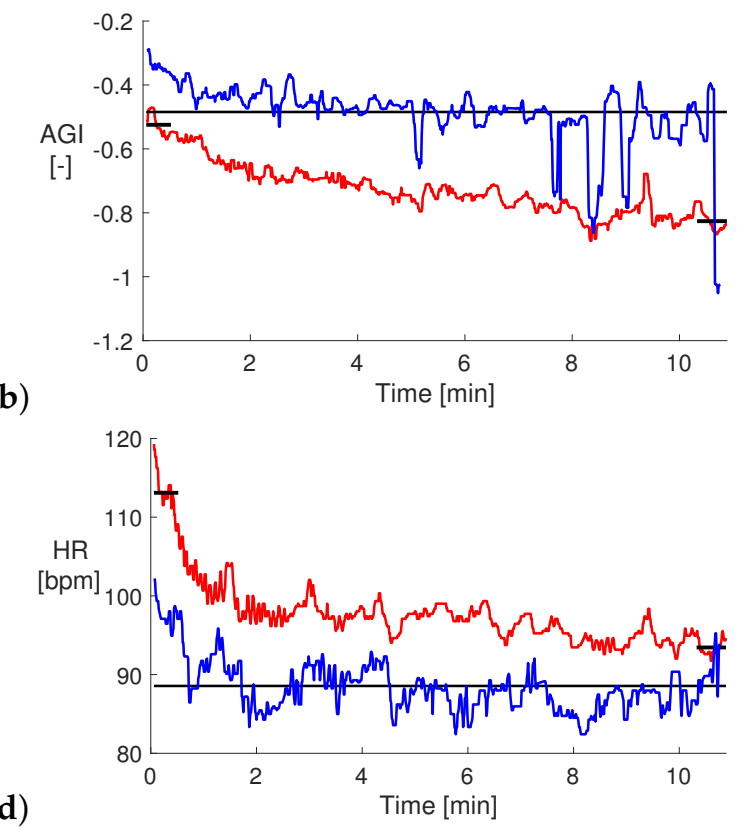

Figure 2. Exemplary Changes in VRR Indices After Exercise: (a) duration of systole $\left(t_{s y s}\right)$; (b) ageing index (AGI); (c) augmentation index (AI); (d) heart rate (HR). Red lines indicate the recovery recording, blue lines the rest recording prior to exercise, and black lines show the mean VRR values during the initial and final parts of the recovery recording and the baseline mean during the rest recording. bpm: beats per minute; au: arbitrary units 


\subsection{The Consistency of Changes in VRR Indices Between Subjects}

Figure 3 shows the number of subjects who exhibited a significant change in each VRR index in a particular direction after exercise. Several indices changed in a consistent direction for most subjects. These included indices calculated from the timings (indicated by $t$ ) and second derivative of the pulse wave (e.g., $e / a, c / a$ and AGI). For instance, the duration of systole $\left(t_{s y s}\right)$ increased during recovery for all 39 subjects. Similarly, the systolic pulse wave area $(A 1)$ and several timing measurements all increased in most subjects, and the HR decreased as observed previously [4].

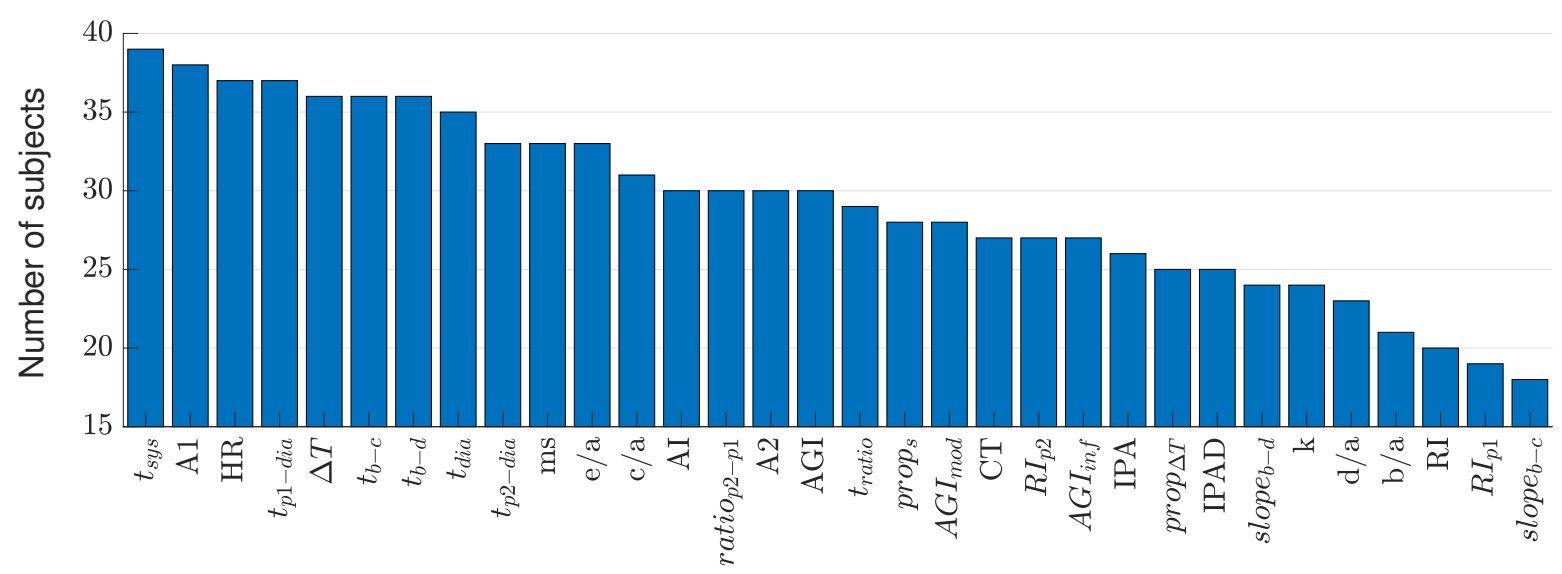

Figure 3. The number of subjects (out of 39) who exhibited a significant change in each VRR index in a particular direction after exercise. Indices are defined in the Abbreviations section (see end).

\subsection{The Rate of Changes in VRR Indices}

Figure 4 shows the percentage change in VRR indices per minute during recovery from exercise. There was a wide variety in the rate at which different indices changed after exercise. This indicates that different indices may be influenced by different cardiovascular properties and may therefore contain complementary information on the state of the vasculature. In addition, the rate of change of some indices differed substantially between different subjects (e.g., $\Delta T$ ), whereas others showed similar rates of change between subjects (e.g., $t_{s y s}$ ). This indicates that the changes in indices may be indicative of subject-specific recovery from exercise and may therefore be useful for stratifying subjects.

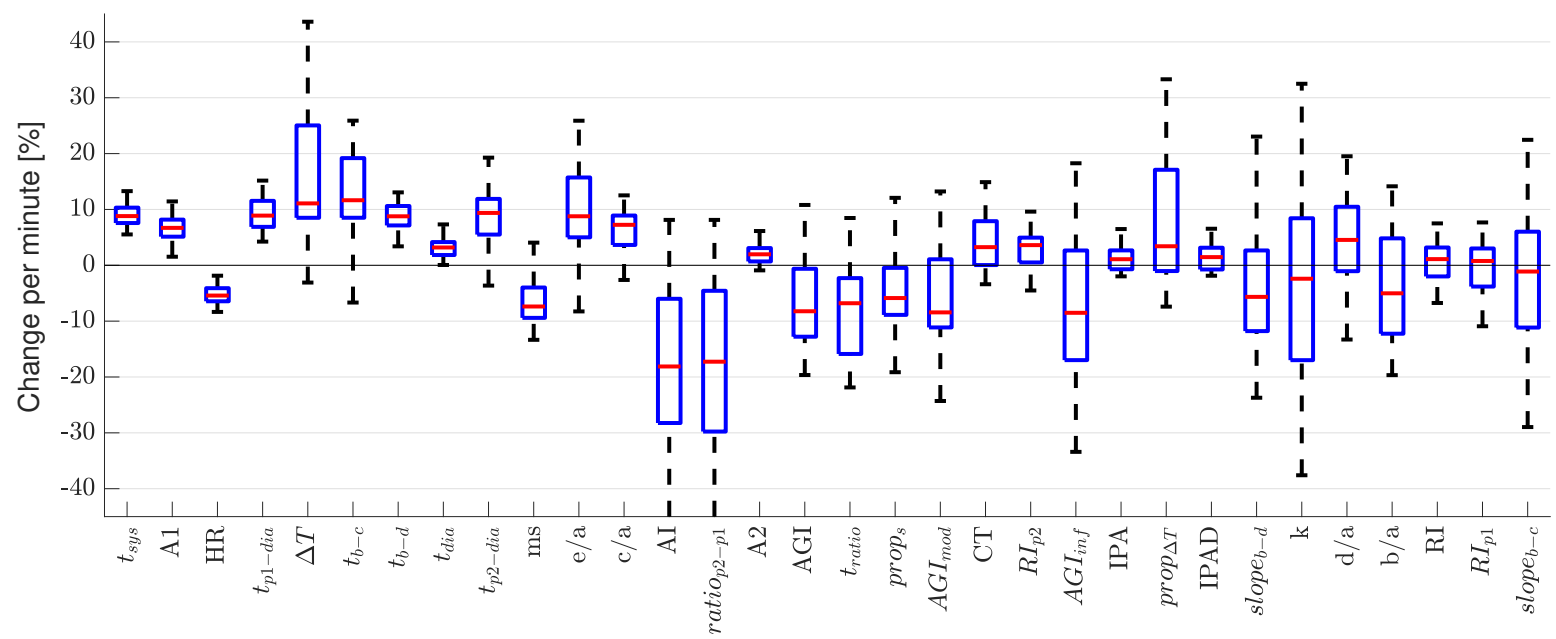

Figure 4. The distributions of the percentage change in each VRR index per minute during recovery from exercise. Boxplots show the median, lower, and upper quartiles. 


\subsection{Limitations and Future Work}

Firstly, the dataset used in this study was acquired from a relatively homogeneous group of healthy subjects. In the future it would be helpful to investigate whether VRR indices change differently after exercise in different groups of subjects (for instance with different levels of health or fitness). This would allow one to assess the potential utility of VRR indices for clinical decision support. Secondly, the post-exercise recordings were acquired shortly after exercise ceased (after time for subjects to move from the treadmill to the bed), and for approximately $10 \mathrm{~min}$. In the future it would be helpful to study recordings from immediately after exercise, and for a longer time period to capture more of the recovery. Thirdly, future work should investigate the mechanisms underlying changes in VRR indices to determine which indices would be most suitable for assessing cardiovascular health.

\subsection{Applications}

We envisage two settings in which VRR could potentially be used: in daily life, and in exercise tests. If the approach was implemented in smart wearables (e.g., fitness bands), then individuals could measure their VRR in routine activities, such as stair climbing, walking, and running. The challenges for this application are that: (i) the activities would not be standardized, so further work would be required to contextualize the VRR according to the level of activity; and (ii) subjects may still be moving after the end of the activity, impairing PPG signal quality. The Parkrun initiative provides a convenient, relatively standardized, weekly exercise regime [11] which could allow self-assessment of VRR. If VRR is found to give clinically useful information then it may be useful to measure it in exercise tests, potentially providing additional insight into the body's ability to recover from exercise.

\section{Conclusions}

This study demonstrated the feasibility of extracting VRR indices from the PPG signal, which is routinely acquired by smart wearables. VRR indices which exhibited consistent inter-subject changes after exercise were identified as candidates for future research (namely those extracted from pulse wave timings and from its second derivative). We observed that the rates of changes in VRR indices differed between indices, and between subjects. This indicates that different indices may be influenced by different physiological mechanisms, and that the recovery may be subject-specific. Therefore, further work should investigate the physiological origins of changes in VRR indices, and determine whether they could be used to usefully assess cardiovascular health in both clinical settings and everyday life.

Author Contributions: conceptualization, P.H.C.; methodology, P.H.C., D.H. and J.A.; software, D.H. and P.H.C.; validation, D.H. and P.H.C.; formal analysis, D.H. and P.H.C.; investigation, D.H. and P.H.C.; resources, P.H.C. and J.A.; data curation, D.H. and P.H.C.; writing-original draft preparation, D.H. and P.H.C.; writing-review and editing, J.A, D.H. and P.H.C.; visualization, D.H., P.H.C. and J.A.; supervision, P.H.C. and J.A.; project administration, P.H.C. and J.A.; funding acquisition, P.H.C..

Funding: This research was funded by the British Heart Foundation (BHF), grant number [PG/15/104/31913], and a Research Experience Placement grant awarded to Peter Charlton by the London Interdisciplinary Doctoral Programme (LIDo). It was additionally supported by the Wellcome/EPSRC Centre for Medical Engineering at King's College London, grant number [WT 203148/Z/16/Z]. The views expressed are those of the authors and not necessarily those of the BHF, LIDo, Wellcome Trust, or EPSRC.

Conflicts of Interest: The authors declare no conflict of interest. The funders had no role in the design of the study; in the collection, analyses, or interpretation of data; in the writing of the manuscript, or in the decision to publish the results.

Data Access Statement: The data used in this research are available subject to the terms of access, as described at http:/ / peterhcharlton.github.io/RRest. Further information about the data and conditions of access can be found by emailing research.data@kcl.ac.uk. 


\section{Abbreviations}

The following abbreviations are used to denote the VRR indices. Further explanation is provided in [10] (Table 3).

\begin{tabular}{|c|c|}
\hline$t_{\text {sys }}$ & duration of systole \\
\hline$A 1, A 2$ & systolic, diastolic area \\
\hline$H R$ & heart rate \\
\hline$t_{p 1-d i a}$ & time between $p 1$ and dia \\
\hline$\Delta T$ & time between $s$ and dia \\
\hline$t_{b-c}$ & time between $b$ and $c$ \\
\hline$t_{b-d}$ & time between $b$ and $d$ \\
\hline$t_{d i a}$ & duration of diastole \\
\hline$t_{p 2-d i a}$ & time between $p 2$ and dia \\
\hline ms & maximum slope \\
\hline$e / a$ & amplitude of $e$ divided by that of $a$ \\
\hline$c / a$ & amplitude of $c$ divided by that of $a$ \\
\hline$A I$ & augmentation index \\
\hline ratio $_{p 2-p 1}$ & amplitude of $p 2$ divided by $p 1$ \\
\hline$A G I, A G I_{\text {mod }}$ & (modified) ageing index \\
\hline$t_{\text {ratio }}$ & time of $s$ divided by time of time of $d i c$ \\
\hline props $_{s}$ & time of $s$ divided by pulse wave duration \\
\hline$R I_{p 1}$ & reflection index calculated using $p 1$ \\
\hline$C T$ & time of $s$ \\
\hline$R I_{p 2}$ & reflection index calculated using $p 2$ \\
\hline$A G I_{\text {inf }}$ & informal ageing index \\
\hline$I P A$ & $A 2 / A 1$ \\
\hline $\operatorname{prop}_{\Delta T}$ & $\Delta T$ divided by duration of pulse wave \\
\hline$I P A D$ & $I P A=d / a$ \\
\hline slope $_{b-d}$, slope $_{b-c}$ & slope from $b$ to $d$, or $c$ \\
\hline$k$ & spring constant \\
\hline$d / a$ & amplitude of $d$ divided by that of $a$ \\
\hline$b / a$ & amplitude of $b$ divided by that of $a$ \\
\hline RI & reflection index calculated using $s$ \\
\hline
\end{tabular}

\section{References}

1. Qiu, S.; Cai, X.; Sun, Z.; Li, L.; Zuegel, M.; Steinacker, J.M.; Schumann, U. Heart rate recovery and risk of cardiovascular events and all-cause mortality: A meta-analysis of prospective cohort studies. J. Am. Heart Assoc. 2017, 6, doi:10.1161/JAHA.117.005505.

2. Guazzi, M.; Arena, R.; Halle, M.; Piepoli, M.F.; Myers, J.; Lavie, C.J. 2016 focused update: Clinical recommendations for cardiopulmonary exercise testing data assessment in specific patient populations. Eur. Heart J. 2018, 39, 1144-1161, doi:10.1093/eurheartj/ehw180.

3. Zhang, Z.; Pi, Z.; Liu, B. TROIKA: A general framework for heart rate monitoring using wrist-type photoplethysmographic signals during intensive physical exercise. IEEE Trans. Biomed. Eng. 2015, 62, 522-531, doi:10.1109/TBME.2014.2359372.

4. Mutter, A.F.; Cooke, A.B.; Saleh, O.; Gomez, Y.H.; Daskalopoulou, S.S. A systematic review on the effect of acute aerobic exercise on arterial stiffness reveals a differential response in the upper and lower arterial segments. Hypertens. Res. 2017, 40, 146-172, doi:10.1038/hr.2016.111.

5. Munir, S.; Jiang, B.; Guilcher, A.; Brett, S.; Redwood, S.; Marber, M.; Chowienczyk, P. Exercise reduces arterial pressure augmentation through vasodilation of muscular arteries in humans. Am. J. Physiol. Heart Circ. Physiol. 2008, 294, H1645-H1650, doi:10.1152/ajpheart.01171.2007.

6. Wang, A.; Yang, L.; Wen, W.; Zhang, S.; Hao, D.; Khalid, S.G.; Zheng, D. Quantification of radial arterial pulse characteristics change during exercise and recovery. J. Physiol. Sci. 2018, 68, 113-120, doi:10.1007/s12576-016-0515-7. 
7. Charlton, P.H.; Bonnici, T.; Tarassenko, L.; Clifton, D.A.; Beale, R.; Watkinson, P.J. An assessment of algorithms to estimate respiratory rate from the electrocardiogram and photoplethysmogram. Phys. Meas. 2016, 37, 610-626, doi:10.1088/0967-3334/37/4/610.

8. Orphanidou, C.; Bonnici, T.; Charlton, P.; Clifton, D.; Vallance, D.; Tarassenko, L. Signal-quality indices for the electrocardiogram and photoplethysmogram: Derivation and applications to wireless monitoring. IEEE J. Biomed. Health Inform. 2015, 19, 832-838, doi:10.1109/JBHI.2014.2338351.

9. Karlen, W.; Ansermino, J.M.; Dumont, G. Adaptive pulse segmentation and artifact detection in photoplethysmography for mobile applications. In Proceedings of the 2012 Annual International Conference of the IEEE Engineering in Medicine and Biology Society, San Diego, CA, USA, 28 August-1 September 2012; doi:10.1109/EMBC.2012.6346628.

10. Charlton, P.H.; Celka, P.; Farukh, B.; Chowienczyk, P.; Alastruey, J. Assessing mental stress from the photoplethysmogram: A numerical study. Phys. Meas. 2018, 39, 054001, doi:10.1088/1361-6579/aabe6a.

11. Reece, L.J.; Quirk, H.; Wellington, C.; Haake, S.J.; Wilson, F. Bright Spots, physical activity investments that work: Parkrun; a global initiative striving for healthier and happier communities. Br. J. Sports Med. 2018, doi:10.1136/bjsports-2018-100041.

(C) 2018 by the authors. Licensee MDPI, Basel, Switzerland. This article is an open access article distributed under the terms and conditions of the Creative Commons Attribution (CC BY) license (http://creativecommons.org/licenses/by/4.0/). 